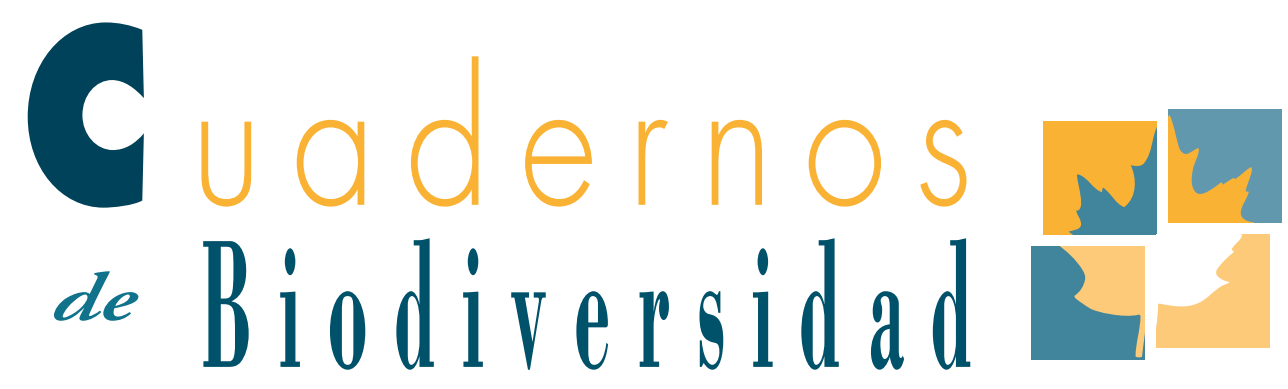

Cuadernos de Biodiversidad 62 (2022): 14-25

ISSN: 2254-6I $2 \mathrm{X}$

DOI:IO.I4I $98 /$ CDBIO.2022.62.02

\title{
Biodiversidad de sírfidos (Diptera, Syrphidae) y su relación con las plantas del campus de la Universidad de Alicante (España)
}

\section{Biodiversity of syrphids (Diptera, Syrphidae) and their relationship with plants on the campus of the University of Alicante (Spain)}

\section{Álvaro Conca Esquembre*, Ma Ángeles Marcos García ${ }^{\mathrm{I}}$}

I Instituto de Investigación CIBIO,

Universidad de Alicante, España

alvaroconcaesquembre@gmail.com

marcos@ua.es

*AUTOR DE CORRESPONDENCIA

\section{iD Álvaro Conca ESQUEMBRE}

iD Ma Ángeles Marcos GARCía

Recibido: 3/07/2021

Aceptado: 16/01/2022

Publicado: 27/01/2022

(C) 2022 Álvaro Conca Esquembre, Ma Ángeles Marcos García LICENCIA:

Este trabajo se publica bajo una Licencia Creative Commons Reconocimiento 4.0 Internacional.

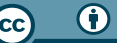

\section{Cómo CITAR:}

Conca Esquembre, A., Marcos García, M.A. (2022). Biodiversidad de sírfidos (Diptera, Syrphidae) y su relación con las plantas del campus de la Universidad de Alicante (España). Cuadernos de Biodiversidad (62), 14-25. https://doi. org/10.14198/cdbio.2022.62.02

\section{RESUMEN}

Aproximadamente, el 35\% de las especies de sírfidos (Diptera, Syrphidae) que se conocen presentan larvas de hábitos depredadores, desempeńando en la naturaleza una importante función como controladores de plagas. Por otro lado, los adultos se alimentan de polen y néctar, por lo que participan activamente en el proceso de la polinización. En este estudio, se ha registrado la presencia de especies de esta familia de dípteros en el campus de la Universidad de Alicante, centrándonos principalmente en las especies depredadoras por su potencial en el control natural de plagas. El registro de las especies vegetales de las zonas verdes y ajardinadas del campus en las que se ha observado la presencia de huevos, larvas, pupas o adultos de sírfidos sobre sus flores, ha permitido establecer una red de interacciones biológicas sírfidoplanta en el campus universitario. Los resultados sugieren que la biodiversidad de plantas silvestres, ruderales o espontáneas que crecen en el campus, algunas consideradas como malas hierbas, favorece el mantenimiento de las poblaciones de sírfidos. Se registraron un total de 27 especies de sírfidos. El mayor número de especies se encontraron sobre especies vegetales pertenecientes a la familia Euphorbiaceae y Compositae. El néctar y polen de las flores de estas especies vegetales representan excelentes recursos tróficos para los adultos de los sírfidos y, por otra parte, estas plantas actúan de reservorio y potenciación de las larvas de especies de sírfidos depredadores que también controlan plagas de las plantas ornamentales del campus.

Palabras clave: Syrphidae; enemigos naturales; polinización; malas hierbas; plantas ornamentales; conservación.

\section{ABSTRACT}

Approximately 35\% of the known species of syrphids (Diptera, Syrphidae) have larvae with predatory habits, playing an important role in nature as pest controllers. On the other hand, adults feed on pollen and nectar, so they actively participate in the pollination process. In this study we have recorded the presence of species of this family of Diptera on the campus of the University of Alicante, focusing mainly on predatory species for their potential in natural pest control. The registration of plant species in green and landscaped areas of the campus in which the presence of syrphid eggs, larvae, pupae or adults on their flowers has been observed, has allowed us to establish a network of biological interactions syrphidplant on the university campus. The results suggest that the biodiversity of wild, ruderal or spontaneous plants growing on the campus, some of which are considered weeds, favors the maintenance of syrphid populations. A total of 27 species of syrphids were recorded. The greatest number of species were found on plant species belonging to the Euphorbiaceae and Compositae families. The nectar and pollen from the flowers of these plant species represent excellent trophic resources for the adults of the syrphids and, on the other hand, these plants act as a reservoir for and enhance the larvae of predatory syrphid species that also control pests of ornamental plants on the campus.

Key words: Syrphidae; natural enemies; pollination; wild plants; ornamental plants; conservation. 


\section{INTRODUCCIÓN}

El uso de fitosanitarios para el control de plagas produce a medio y largo plazo la contaminación de aguas y suelos, la aparición de plagas secundarias, de nuevas resistencias a los biocidas y la muerte masiva e indiscriminada de muchas especies de animales, sobre los que no va dirigida la acción de control (Hedo, 2012). Una buena parte de las especies de insectos que son afectadas negativamente por el uso de biocidas, son beneficiosas porque realizan importantes funciones ecosistémicas como es el caso de los polinizadores y de los enemigos naturales de las plagas (Morales et al., 2006). Los sírfidos (Diptera, Syrphidae) son insectos muy necesarios en los ecosistemas porque realizan estas dos funciones ecosistémicas. En la actualidad, gracias a la Normativa Comunitaria y Nacional, progresivamente se va reduciendo el uso de productos químicos de síntesis y sustituyéndolos por estrategias de control biológico e integrado, que además de ser respetuosos con la biodiversidad, representan una significativa mejora en la salud humana.

Una de las alternativas actuales para el control de plagas se basa en el uso de enemigos naturales que controlan las especies plagas y a los vectores de enfermedades. Se trata del Control Biológico mediante el manejo o provisión de recursos favorables para las poblaciones de depredadores o parasitoides de las especies que causan plagas.

Para poder establecer medidas acertadas de control biológico, es imprescindible conocer los ciclos reproductivos, biología y ecología de la especie causante de la plaga, así como las relaciones que mantienen con sus agentes biológicos de control. Una vez identificados sus requerimientos, se deben seleccionar los enemigos naturales más adecuados para proceder al control de la plaga hasta que su presencia no represente un perjuicio económico, sanitario o ambiental (Morales et al., 2006). Cuando el número de enemigos naturales en condiciones naturales es insuficiente y no se logra el control de la plaga, hay que recurrir a su liberación, bien de manera preventiva (antes de que llegue la plaga), bien curativa una vez se constata el daño. Estos agentes biológicos beneficiosos son criados masivamente en biofábricas y cada una de las especies comercializadas tiene establecido un protocolo de actuación específico para su uso en el control de plagas.

Siempre que sea posible en el control biológico de plagas, conviene liberar especies autóctonas, no sólo por evitar introducir especies exóticas que pueden convertirse en potenciales plagas y ocasionar nuevos desequilibrios medioambientales, sino también porque las especies autóctonas son las que mejor van a establecerse en el medio y a ejercer mejor el control de la plaga, al estar adaptadas a ese ecosistema y a sus condiciones bioclimáticas. Por este motivo, es importante conocer cuáles son las especies autóctonas que ejercen un control natural en nuestros ecosistemas, también en los jardines urbanos.

La vegetación del campus de la Universidad de Alicante está constituida por especies de plantas ornamentales seleccionadas por sus cualidades estéticas, actuando además alguna de ellas como lugar de refugio para la denominada "entomofauna útil" y albergan una cierta biodiversidad que colabora en funciones ecosistémicas tan importantes como el control de plagas y la polinización.

Los sírfidos, conocidos como moscas de las flores ("flowerflies") por su frecuente visita floral o moscas cernidoras ("hoverflies") por su característico vuelo estático (Hurtado, 2013). La característica morfológica principal que define este grupo de dípteros es la venación alar, presentando nervios transversales que se unen a los radiales formando las celdas discal y radial posterior y además un nervio vestigial, la vena espuria que recorre la celda basal y la discal (Van Veen, 2004). Además, por la heterogeneidad de los hábitats en los que se desarrollan sus larvas, los sírfidos son una herramienta de estudio muy útil como bioindicadores de la calidad de los ecosistemas en los que viven (Sommaggio, 1999).

Las funciones ecosistémicas más destacables que realizan los sírfidos son el control biológico de plagas y la polinización. En su fase larvaria presentan gran variedad de hábitos de vida: saprófagos, fitófagos (algunas especies se alimentan de bulbos de Liliáceas y Amarillidáceas), saproxílicos, micófagos y depredadores (Rotheray \& Gilbert, 2011). Por otra parte, los adultos de todas las especies presentan hábitos florícolas, ya que se alimentan de polen y néctar que 
necesitan para madurar sexualmente y para realizar ese vuelo sostenido que les caracteriza, lo que les convierte en importantes agentes polinizadores.

Solo unas pocas especies depredadoras están especializadas respecto al tipo de presa, alimentándose la mayor parte de ellas, de áfidos de muchas especies. El ciclo completo de las especies depredadoras dura entre una y tres semanas (Pineda \& Marcos-García, 2008), aunque las variables ambientales y la disponibilidad de presas, alteran la duración de estos ciclos, alargándose cuando la disponibilidad de alimento es escasa y cuando las temperaturas son más bajas (Auad, 2003).

Los registros de ejemplares adultos de sírfidos en el campus empiezan en marzo con la llegada de la primavera, momento en el que suben las temperaturas y comienza la floración en los espacios verdes del campus, que concentran la mayor biodiversidad de especies vegetales y como consecuencia, de sus insectos asociados. El incremento de las temperaturas y la aparición de los primeros brotes vegetales provoca la aparición de las especies fitófagas de estas plantas, provocando en algunos casos el aumento rápido en las poblaciones de áfidos que son el principal recurso trófico de los sírfidos depredadores. No obstante, una mayor abundancia de flores, no siempre se traduce en un incremento en el número de insectos beneficiosos. Su relación con las plantas depende del color, forma, porcentaje y composición de azúcares en el néctar, accesibilidad al polen, etc. (Mexzón \& Chinchilla, 2003) e incluso de los tratamientos que se hayan podido aplicar a la vegetación de la zona (plaguicidas, biocidas, etc.).

En este trabajo se ha estudiado la biodiversidad y la fenología de las especies autóctonas de sírfidos que visitan o se desarrollan en las plantas ornamentales y espontáneas (algunas consideradas malas hierbas) del campus de la Universidad de Alicante (UA). Para aquellas especies de hábitos larvarios depredadores, se aportan datos sobre las interacciones biológicas que se establecen entre los tres niveles tróficos considerados (planta-fitófago-enemigo natural) con el objetivo de aportar información que ayude a la mejora de las estrategias de control biológico e integrado de plagas.

\section{METODOLOGÍA}

El estudio ha sido realizado en el campus universitario de la Universidad de Alicante (San Vicente del Raspeig, 38²3'15" N 0³0' 47" W, 80 ha de extensión), tanto en las zonas ajardinadas, como en parcelas intercaladas con vegetación silvestre. El campus se ubica en el piso bioclimático termomediterráneo, de ombroclima semiárido y con una temperatura media anual de $17,7^{\circ} \mathrm{C}$ (Costa, 1982). Las condiciones climáticas de la zona donde se ha realizado el estudio destacan por tener temperaturas suaves casi todo el año, veranos muy calurosos y lluvias escasas (Herrera, 1984).

Se observan dentro del campus especies arbustivas ornamentales propias de clima mediterráneo (Nerium oleander, Pistacea lentiscus, Acanthus mollis, etc.), árboles frutales típicos del clima mediterráneo (Citrus reticulata, Punica granatum, Olea europaea, Ceratonia siliqua, etc.), especies ornamentales no autóctonas (Ficus spp., Tipuana sp., Bougainvillea sp., Hibiscus rosa-sinensis, etc.), además de las especies hortícolas de un pequeño huerto ecológico situado en su interior (Cynara scolymus, Vicia faba, Solanum lycopersicum, Lactuca sativa, etc.) y de especies ruderales y adventicias. Se han registrado, en el campus de la Universidad de Alicante, al menos 407 taxones pertenecientes a 241 géneros de 52 familias botánicas diferentes, representando esta cifra el $54 \%$ de las especies totales descritas de malas hierbas en España (Brotons et al., 2009).

Para el estudio de los sírfidos, se han seguido dos métodos tradicionales de muestreo: la recolección de individuos adultos mediante el uso de la manga entomológica y la búsqueda de las fases inmaduras de sírfidos sobre las plantas para su cría en el laboratorio. La captura de adultos mediante la manga entomológica nos permite conocer la relación del sírfido con la planta de la que se alimentan los adultos o en la que se realiza la oviposición y la búsqueda de fases inmaduras sobre las plantas, nos permite relacionar las especies de sírfidos con sus presas y la planta huésped donde se desarrollan.

Los puntos de muestreo se seleccionaron teniendo en cuenta la mayor presencia de flores y de plantas 
silvestres u ornamentales en las que se pueden encontrar ejemplares inmaduros de sírfidos. En total, se han estudiado más de 30 puntos de muestreo en el campus de la Universidad de Alicante (Figura 1) coincidiendo con zonas ajardinadas con vegetación ornamental, un huerto ecológico (Figura 2) y flora silvestre establecida de manera espontánea a lo largo de todo el campus.

Se realizaron 4 o 5 muestreos semanales con una duración mínima de 60 minutos cada uno en cada zona de muestreo establecida, con una media de $3 \mathrm{~h}$ diarias. Como se trata de insectos heliófilos, la actividad de campo se desarrolló exclusivamente en horarios diurnos y en días soleados, entre las 9:00h y 13:00h para abarcar el periodo de mayor actividad de los sírfidos en todos los puntos. Asimismo, se descartaron los días lluviosos o con fuertes rachas de viento.

La manga empleada presenta un aro metálico de $35 \mathrm{~cm}$ de diámetro y una malla blanca de luz muy fina (1 mm o inferior) (Figura 3). La captura de sírfidos se realizó al vuelo o posados sobre las flores o sobre la vegetación silvestre. Tras la recolección, los imagos se introdujeron en pequeños

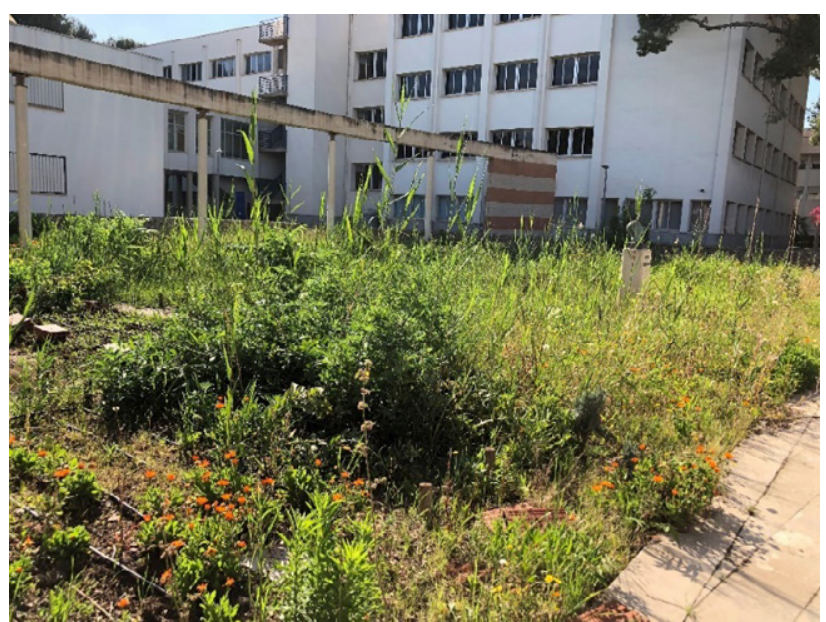

Figura 2: Huerto ecológico Universidad de Alicante 2021 (Foto: M.A. Marcos García).

tubos herméticos etiquetados con la información necesaria (ubicación, fecha, hora y planta) para su identificación y posterior análisis.

El muestreo de los estados inmaduros de los sírfidos (huevos, larvas y pupas) se realizó con un pincel fino, recogiendo además la planta huésped y una muestra del insecto fitófago presa. Estas capturas se hicieron dos veces a la semana. Las muestras se

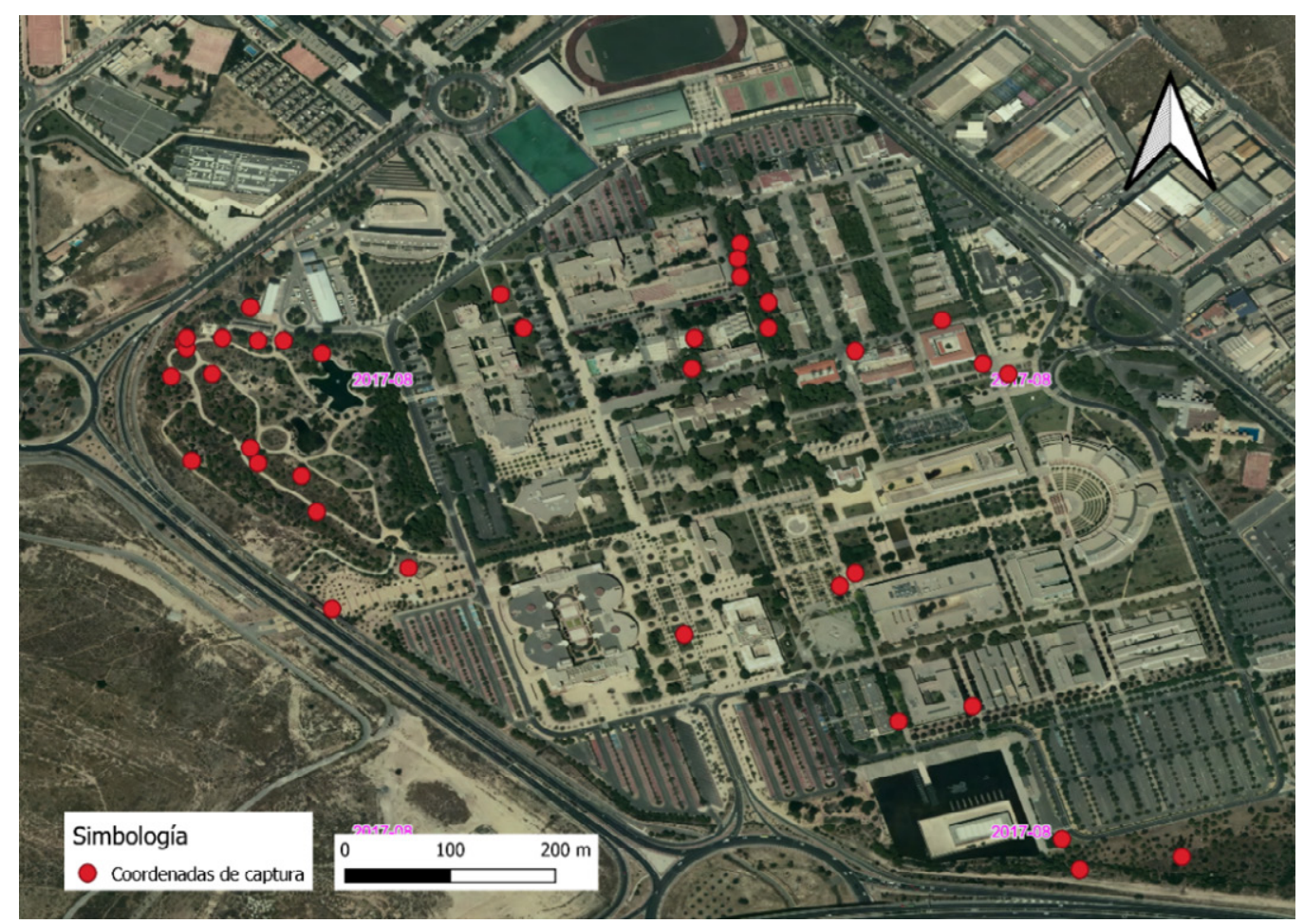

Figura 1: Mapa con los puntos donde se han realizado capturas de sírfidos. Elaboración propia a partir de QGIS . 


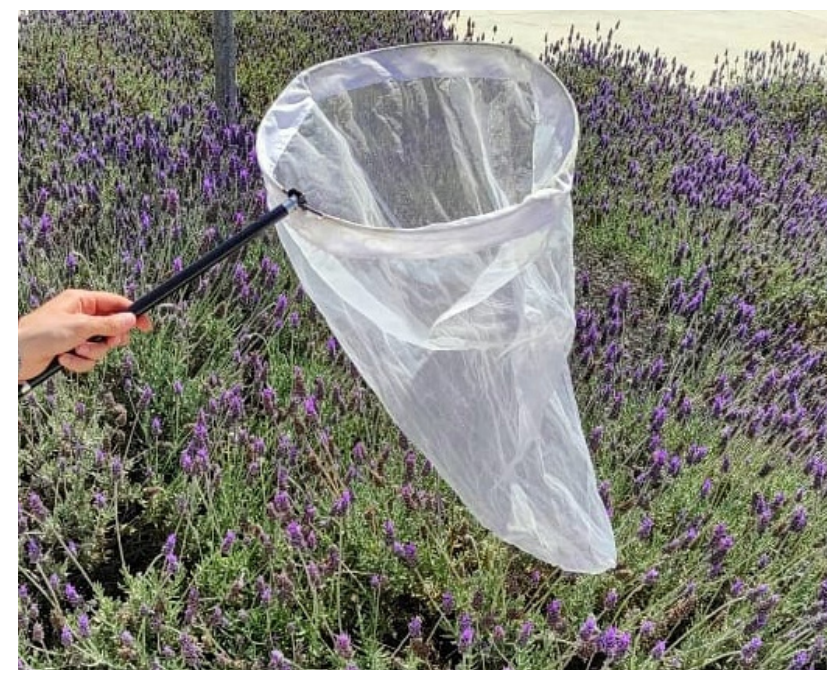

Figura 3: Manga entomológica utilizada para la captura (Foto: Álvaro Conca).

llevaron al laboratorio para su cría y obtención de los adultos que permite una identificación rigurosa a nivel específico. Siempre que ha sido posible, las especies vegetales fueron identificadas mediante fotos para evitar su extracción. De esta forma, se puede conocer la identidad del sírfido, la duración de su ciclo biológico y las asociaciones sírfido-planta huésped-áfido presa.

En el laboratorio, los sírfidos capturados fueron sacrificados sometiéndolos a $-4^{\circ} \mathrm{C}$ en un congelador durante 24 horas. Para su preparación y conservación se ha seguido la metodología propia para este grupo de insectos (Marcos-García \& Galante, 2004), secándose las muestras en una estufa a $40^{\circ} \mathrm{C}$ durante siete días para evitar la aparición de hongos u otros microorganismos que puedan estropear o contaminar la muestra (Luna, 2005). Una vez el ejemplar está seco, se procedió al etiquetado y colocación en cajas y armarios entomológicos herméticos y alojados en estancias apropiadas con baja humedad y $18^{\circ} \mathrm{C}$ de temperatura. Las muestras se encuentran depositadas en la Colección Entomológica de la Universidad de Alicante (CEUA-CIBIO).

Los estados preimaginales fueron mantenidos individualmente en un ambiente controlado, en recipientes de $15 \mathrm{ml}$, con una malla de poro fino que permite la aireación, con una temperatura de $27 \pm$
$2{ }^{\circ} \mathrm{C}$, humedad relativa del $70 \pm 10 \%$ y un fotoperiodo de 14:10 h. Las larvas fueron alimentadas diariamente ad libitum con áfidos hasta completar su ciclo biológico. La recolecta de pulgón que sirvió de alimento para los estados inmaduros se hizo mediante la extracción de brotes/ramas de la misma especie de planta en la que se encontraron y mantenidos vivos en recipientes. Los imagos obtenidos fueron procesados de acuerdo con la metodología anteriormente descrita y se depositaron en la Colección CEUA-CIBIO.

La identificación de las especies de sírfidos ha sido realizada con la ayuda de un microscopio estereoscópico (Leika MZ95) con iluminación incorporada. Algunos géneros como Paragus Latreille, 1804 o Eumerus (Meigen, 1882) se identificaron a nivel de especie mediante la extracción y estudio de la genitalia de los machos, cuya estructura asegura una identificación precisa. Para la identificación de las especies se ha seguido a Van Veen (2004) con la ayuda de bibliografía específica de algunos géneros. Los áfidos a su vez fueron identificados por especialistas afidólogos mediante fotografías hechas en el momento de su recogida o en el laboratorio, siempre acompañado de la información de la planta huésped en la que se recogió cada ejemplar.

La información sobre plantas adventicias, así como las ornamentales, se obtuvo a partir de expertos botánicos y con ayuda de la información recopilada en Brotons et al. (2009).

Los mapas de interacciones biológicas entre los tres componentes del estudio, depredador (sírfido)plaga (áfidos)-planta huésped, se han realizado con el programa R Project.

Durante el periodo del estudio se produjeron diferentes labores de jardinería, el uso de tratamientos químicos y la eliminación de flora silvestre adventicia que repercutieron negativamente en las capturas, cría y alimentación de los estados inmaduros de los sírfidos. Por este motivo, el muestreo no pudo mantener su homogeneidad en todos los puntos y obligó a mantener en el laboratorio una pequeña cría de pulgón (Rhopalosyphum padi (Linnaeus, 1758) sobre cebada que permitiera la cría de los inmaduros. 


\section{RESULTADOS}

Se registró un total de 27 especies de sírfidos, de las cuales, 19 son depredadores de áfidos plaga durante su desarrollo larvario (Tabla 1). Se observa a Episyrphus balteatus (De Geer, 1776) como la más abundante (13,41\%), seguida de Eupeodes corollae (Fabricius, 1794) (11,59\%), siendo ambas especies afidófagas.

Tabla 1: Especies de sírfidos que han sido registradas en estado adulto en el campus de la Universidad de Alicante. También se detallan aquellas especies que se han criado en cautividad desde sus primeros estados larvarios, o desde el huevo, y aquellas de las que se tiene registro bibliográfico en el campus de San Vicente del Raspeig (HernándezMartínez \& Marcos-García, 2019). Leyenda: (h) Especies criadas desde huevo; (l) Especies criadas desde larva; $\mathrm{D}=$ depredadora; $\mathrm{F}=$ fitófaga; $\mathrm{Sx}=$ saproxílica; $\mathrm{Sf}=$ saprófaga.

\begin{tabular}{|c|c|c|c|c|}
\hline Especie & $\begin{array}{l}\text { Ejemplares } \\
\text { adultos } \\
\text { capturados }\end{array}$ & $\begin{array}{l}\text { Ejemplares } \\
\text { criados }\end{array}$ & $\begin{array}{c}\text { Especies } \\
\text { previamente } \\
\text { registradas en } \\
\text { la UA }\end{array}$ & $\begin{array}{l}\text { Alimentación } \\
\text { de las larvas }\end{array}$ \\
\hline Ceriana vespiformis (Latreille, 1804) & 2 & - & - & Sx \\
\hline Chrysotoxum intermedium Meigen, 1882 & 7 & - & - & $\mathrm{D}$ \\
\hline Dasysyrphus albostriatus (Fallen, 1817) & 1 & - & - & $\mathrm{D}$ \\
\hline Epistrophe eligans (Harris, 1780) & 1 & - & - & $\mathrm{D}$ \\
\hline Episyrphus balteatus (de Geer, 1776) & 12 & $10^{(\mathrm{h})}$ & • & $\mathrm{D}$ \\
\hline Eristalinus aeneus (Scopoli, 1763) & 9 & - & - & Sf \\
\hline Eristalinus taeniops (Wiedemann, 1818) & 4 & - & - & Sf \\
\hline Eristalis arbustorum (Linnaeus, 1758) & 10 & - & - & Sf \\
\hline Eristalis tenax (Linnaeus, 1758) & 5 & - & - & Sf \\
\hline Eumerus amoenus Loew, 1848 & 13 & - & - & $\mathrm{F}$ \\
\hline Eupeodes corollae (Fabricius, 1794) & 15 & $5^{(\mathrm{h})}$ & $\bullet$ & $\mathrm{D}$ \\
\hline Heringia heringi (Zetterstedt, 1843) & 1 & - & - & $\mathrm{D}$ \\
\hline Melanostoma mellinum (Linnaeus, 1758) & 4 & - & - & $\mathrm{D}$ \\
\hline Meliscaeva auricollis (Meigen, 1822) & 3 & - & - & $\mathrm{D}$ \\
\hline Myathropa florea (Linnaeus, 1758) & 1 & - & - & Sx \\
\hline Paragus bicolor (Fabricius, 1794) & 2 & - & - & $\mathrm{D}$ \\
\hline Paragus haemorrhous Meigen 1822 & 2 & - & - & $\mathrm{D}$ \\
\hline Paragus quadrifasciatus Meigen, 1882 & 1 & - & - & $\mathrm{D}$ \\
\hline Paragus tibialis (Fallén, 1817) & 8 & $1^{(1)}$ & - & $\mathrm{D}$ \\
\hline Scaeva albomaculata (Macquart, 1842) & 1 & - & - & $\mathrm{D}$ \\
\hline Scaeva dignota (Rondani, 1857) & 2 & $1^{(1)}$ & - & $\mathrm{D}$ \\
\hline Scaeva pyrastri (Linnaeus, 1758) & 2 & $11^{(\mathrm{h})}$ & - & $\mathrm{D}$ \\
\hline Sphaerophoria rueppellii (Wiedemann, 1830) & 1 & $1^{(1)}$ & - & $\mathrm{D}$ \\
\hline Sphaerophoria scripta (Linnaeus, 1758) & 11 & - & - & $\mathrm{D}$ \\
\hline Syritta pipiens (Linnaeus, 1758) & 13 & - & - & Sf \\
\hline Syrphus vitripennis Meigen, 1822 & - & $2^{(\mathrm{h})}$ & - & $\mathrm{D}$ \\
\hline Xanthogramma marginale (Loew, 1854) & 7 & - & - & $\mathrm{D}$ \\
\hline Total especies: 27 & 128 & 31 & 6 & \\
\hline
\end{tabular}




\section{ESPECIES FITÓFAGAS PRESAS DE LOS SÍRFIDOS}

Las especies fitófagas, potenciales plagas, que se han registrado como presa de las larvas de sírfidos, pertenecen al grupo de los pulgones (Hemiptera, Aphididae). En la Tabla 2 se representa su clasificación taxonómica y la planta huésped asociada donde se han capturado.

Tabla 2: Especies de áfidos y sus plantas huésped en la Universidad de Alicante.

\begin{tabular}{l|l}
\hline Género/ Especie & Planta huésped \\
$\begin{array}{l}\text { Aphis euphorbiae } \\
\text { Kaltenbach, 1843 }\end{array}$ & Euphorbia serrata $\mathrm{L}$. \\
\hline Aphis fabae Scopoli, 1763 & Vicia faba $\mathrm{L}$. \\
\hline Aphis gossypi Glover, 1877 & Hibiscus rosa-sinensis $\mathrm{L}$. \\
\hline $\begin{array}{l}\text { Aphis nerii Boyer de } \\
\text { Fonscolombe, 1841 }\end{array}$ & Nerium oleander $\mathrm{L}$. \\
\hline $\begin{array}{l}\text { Aphis punicae Passerini, } \\
\text { 1863 }\end{array}$ & Punica granatum $\mathrm{L}$. \\
\hline $\begin{array}{l}\text { Capitophorus elaegni (Del } \\
\text { Guercio, 1894) }\end{array}$ & Cynara cardunculus $\mathrm{L}$. \\
\hline $\begin{array}{l}\text { Capitophorus elaegni }(\mathrm{Del} \\
\text { Guercio, 1894) }\end{array}$ & Cynara scolimus $\mathrm{L}$. \\
\hline $\begin{array}{l}\text { Hyalopterus pruni } \\
\text { (Geoffroy, 1762) }\end{array}$ & Arundo donax $\mathrm{L}$. \\
\hline $\begin{array}{l}\text { Hyperomyzus sp. (Börner, } \\
\text { 1933) }\end{array}$ & Sonchus oleraceus $\mathrm{L}$. \\
\hline $\begin{array}{l}\text { Myzaphis rosarum } \\
\text { (Kaltenbach,1843) }\end{array}$ & Rosa sp. \\
\hline $\begin{array}{l}\text { Uroleucon sonchi } \\
\text { (Linnaeus, 1767) }\end{array}$ & Sonchus oleraceus $\mathrm{L}$. \\
\hline & Sonchus tenerrimus $\mathrm{L}$. \\
\hline
\end{tabular}

\section{SÍRFIDOS DEPREDADORES}

Respecto a los sírfidos depredadores que constituyen el tercer nivel de la cadena trófica, se han estudiado 7 especies que han sido criadas en labo- ratorio a partir de sus fases inmaduras (huevo, larva o pupa). También se han registrado sus relaciones con las plantas huésped en las que se han encontrado (Figura 4a) y las especies de áfidos presa (Figura 4b).

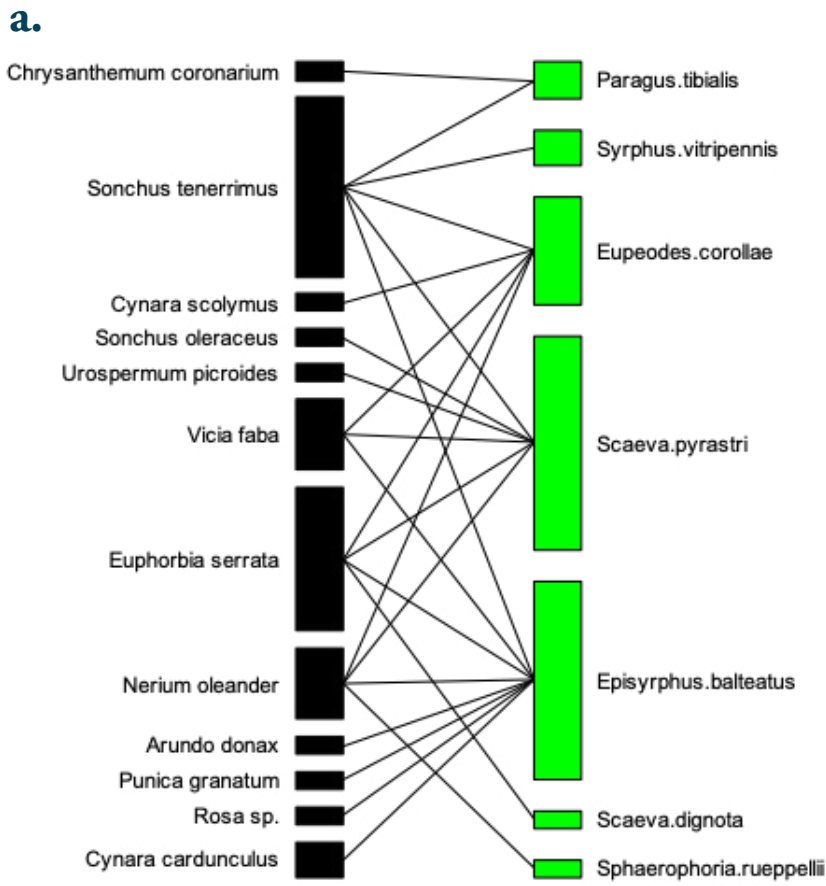

b.

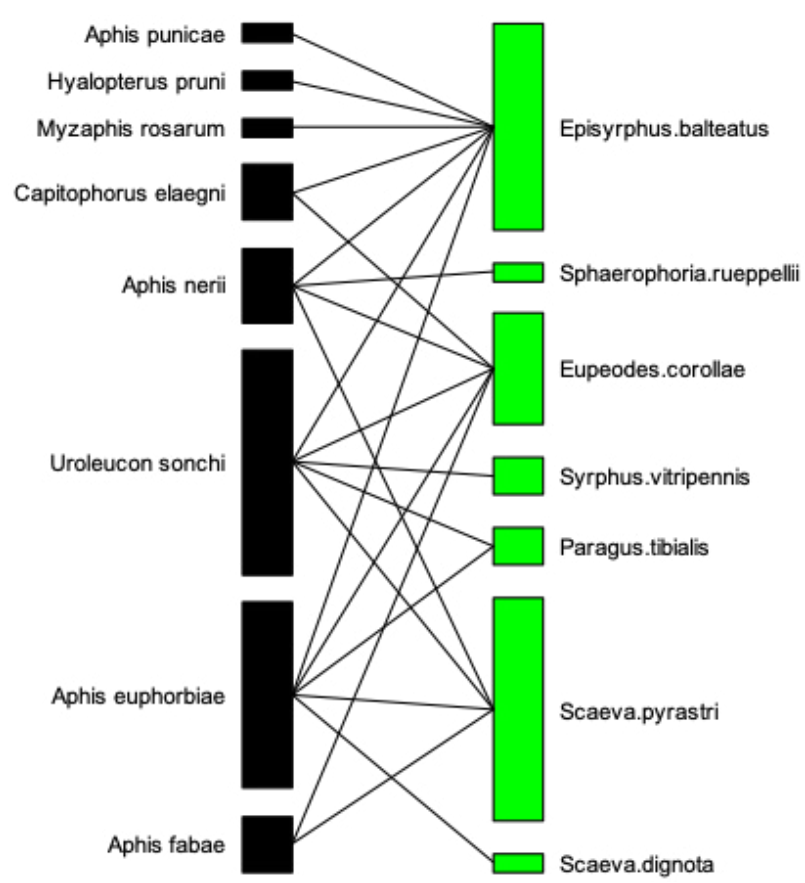

Figura 4: (a) Mapa de interacciones planta huéspedsírfido. (b) Mapa de interacciones áfido presa-sírfido. 
La abundancia de sírfidos adultos a lo largo del día varía temporalmente conforme a la temperatura y el fotoperiodo. Durante los meses de estudio se observa que la actividad de vuelo comienza con las primeras horas de sol, llega a su máximo entre las 11 y las 12 horas solares (Figura 5) y descendiendo paulatinamente hasta la puesta del sol.

Todas las especies de sírfidos registradas, permanecen en el campus al término del periodo de estudio y se constata la presencia y actividad de vuelo de adultos invernantes de algunas especies durante los meses de invierno (febrero y marzo). Se observa además un aumento progresivo en la riqueza de especies a medida que se avanza hacia la primavera (Figura 6). Los datos de los primeros registros de huevos y larvas de las especies depredadoras nos indican el inicio del periodo de maduración, coincidente con el de la aparición de sus presas, las especies plagas, siendo por tanto estos datos de aplicación directa en las futuras estrategias de control biológico de plagas del campus.

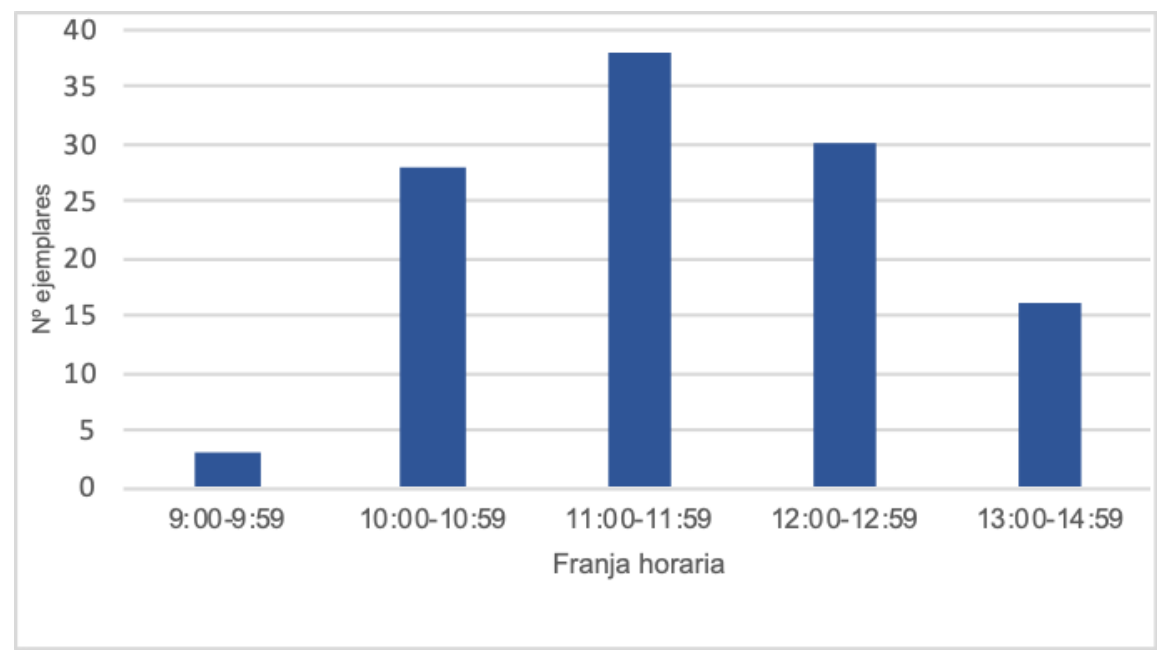

Figura 5: Abundancia acumulada de sírfidos en vuelo en cada franja horaria durante los meses de febrero a abril de 2021.

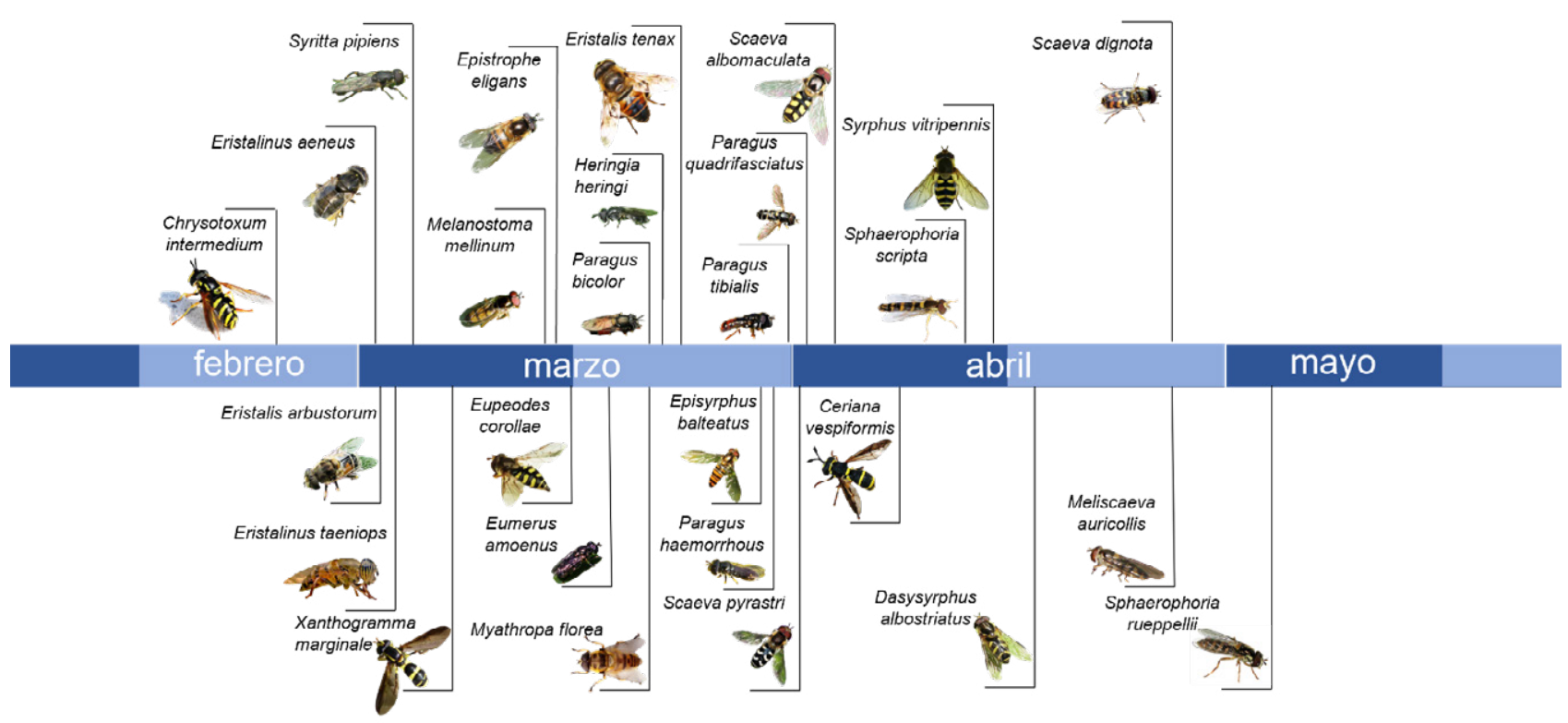

Figura 6: Periodo de actividad de vuelo de las especies de sírfidos capturados en el campus de la UA. El punto en el cual se sitúa la flecha corresponde con el momento en el que se capturó por primera vez un ejemplar de la especie. La línea temporal de cada especie no está marcada por un final ya que se sigue registrando su actividad de vuelo en el campus. 
En el mapa de interacciones de la Figura 7, se reflejan las especies de sírfidos estudiadas y su relación trófica con las plantas del campus. Las interacciones representan ejemplares adultos capturados mientras se alimentaban de polen o néctar, o permanecían en reposo sobre la flor una vez se han alimentado. E. balteatus y E. corollae, parecen ser las dos especies de sírfidos más abundantes en el campus. Ambas junto con Scaeva pyrastri (Linaeus, 1758) son las que mantienen un mayor número de interacciones con las plantas del campus y con las especies de pulgones plaga depredados. Son, por tanto, especies relevantes en la función ecosistémica que ejercen estas tres especies de sírfidos sobre el control natural de las plagas de áfidos del campus. Este patrimonio biológico natural debe preservarse evitando el uso de productos fitosanitarios que reduzcan las poblaciones de estos enemigos naturales cuyos adultos también participan en la polinización.

Las interacciones de las especies depredadoras con sus presas y las plantas huéspedes de éstas, se encuentran representadas en las Figuras $4 \mathrm{a}$ y 4 b, en las que se observa la utilidad del muestreo de fases inmaduras en la vegetación, ya que en el caso de Syrphus vitripennis Meigen, 1882, tanto su presencia en el campus, como el conocimiento de sus relaciones interespecíficas, han podido establecerse gracias a que sus huevos fueron encontrados sobre Sonchus tenerrimus L. y Vicia faba L.

Figura 7: Mapa de interacciones específicas sírfido-planta.

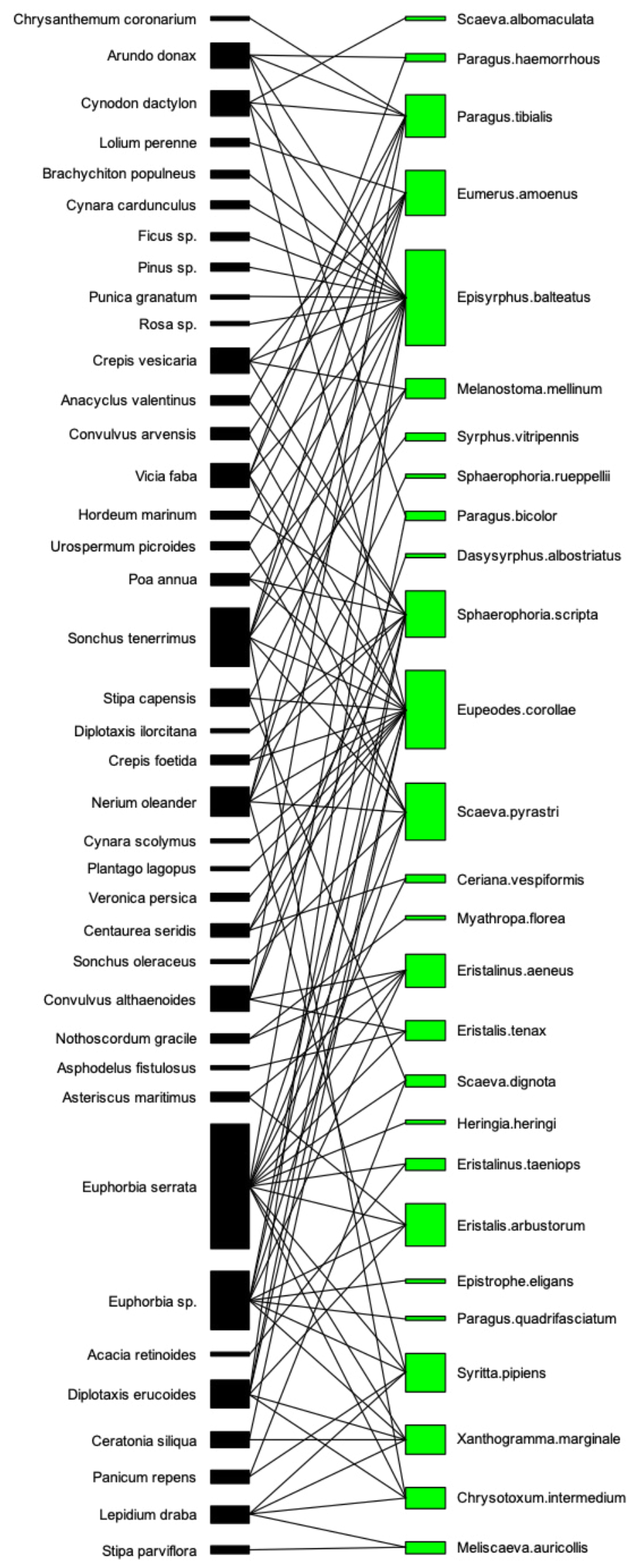


En la Tabla 3 se detallan todas aquellas especies vegetales en las que han sido encontrados huevos, larvas, pupas o adultos de sírfidos, ya sea en especies silvestres (las consideradas malas hierbas), ornamentales o cultivos del huerto en la UA.

\begin{tabular}{|c|c|}
\hline Familia & Especie \\
\hline Alliaceae & $\begin{array}{l}\text { Nothoscordum gracile (Ait.) } \\
\text { Stearn }\end{array}$ \\
\hline Asphodelaceae & Asphodelus fistulosus L. \\
\hline Apocynaceae & Nerium oleander L. \\
\hline \multirow[t]{12}{*}{ Compositae } & Cynara cardunculus L. \\
\hline & Cynara scolymus $\mathrm{L}$. \\
\hline & Anacyclus valentinus $\mathrm{L}$. \\
\hline & Asteriscus maritimus (L.) \\
\hline & Bellis annua $\mathrm{L}$. \\
\hline & Centaurea seridis L. \\
\hline & Chrysanthemum coronarium L. \\
\hline & Crepis foetida L. \\
\hline & Crepis vesicaria $\mathrm{L}$. \\
\hline & Sonchus oleraceus L. \\
\hline & $\begin{array}{l}\text { Sonchus tenerrimus L. subsp. } \\
\text { tenerrimus }\end{array}$ \\
\hline & $\begin{array}{l}\text { Urospermum picroides (L.) Scop. } \\
\text { Ex F. }\end{array}$ \\
\hline \multirow[t]{2}{*}{ Convolvulaceae } & Convolvulus althaeoides L. \\
\hline & Convulvus arvensis $\mathrm{L}$. \\
\hline \multirow[t]{3}{*}{ Cruciferae } & Diplotaxis erucoides (L.) \\
\hline & $\begin{array}{l}\text { Diplotaxis ilorcitana (Sennen) } \\
\text { Aedo \& All. }\end{array}$ \\
\hline & Lepidium draba $\mathrm{L}$. \\
\hline \multirow[t]{2}{*}{ Euphorbiaceae } & Euphorbia serrata L. \\
\hline & Euphorbiasp. \\
\hline \multirow[t]{2}{*}{ Fabaceae } & Vicia faba $\mathrm{L}$. \\
\hline & Ceratonia siliqua $\mathrm{L}$. \\
\hline Lytraceae & Punica granatum L. \\
\hline Moraceae & Ficus sp. \\
\hline Pinaceae & Pinus sp. \\
\hline Plantaginaceae & Plantago lagopus L. \\
\hline \multirow[t]{3}{*}{ Poaceae } & Cynodon dactylon (L.) Pers. \\
\hline & $\begin{array}{l}\text { Hordeum murinum L. subsp. } \\
\text { leporinum }\end{array}$ \\
\hline & Arundo donax $\mathrm{L}$. \\
\hline
\end{tabular}

\begin{tabular}{l|l}
\hline & Lolium perenne $\mathrm{L}$. \\
\hline & Panicum repens $\mathrm{L}$. \\
\hline & Poa annua L. \\
\hline & Stipa capensis Thunb. \\
\hline & Stipa parviflora Desf. \\
\hline Rubiaceae & Veronica persica Poir. \\
\hline Sterculiaceae & $\begin{array}{l}\text { Brachychiton populneus (Scott \& } \\
\text { Endl.) }\end{array}$ \\
\hline Zygophyllaceae & Fagonia cretica $\mathrm{L}$.
\end{tabular}

\section{DISCUSIÓN}

De las 27 especies de sírfidos estudiadas, 19 presentan estados larvarios depredadores y en 7 de estas especies (E. balteatus, E. corollae, Paragus tibialis (Fallén, 1817), Scaeva dignota (Rondani, 1857), S. pyrastri, Sphaerophoria rueppellii (Wiedemann, 1830) y $S$. vitripennis) se ha observado depredación de especies plaga en el campus. Todas ellas son polífagas (Rojo et al., 2003) y se ha constatado que se alimentan de varias especies de áfidos en el campus (Figura 4b). Sus estados inmaduros han sido recogidos en las colonias de pulgones, destacando $E$. balteatus, cuyas larvas se han encontrado depredando hasta 6 especies diferentes de áfidos presentes en las plantas del campus, siendo por lo tanto un buen agente natural de control de áfidos. Los pulgones, a su vez, se han encontrado en diferentes especies vegetales, tanto en plantas silvestres de los géneros Euphorbia sp. y Sonchus sp.; especies hortícolas del huerto ecológico, como Cynara cardunculus L., Cynara scolymus L. y $V$. faba; especies de árboles frutales como $P$. granatum o Citrus spp., o plantas ornamentales como Nerium oleander L., Hibiscus sp. o Rosa sp.

Entre las interacciones sírfido-planta registradas, destacamos las familias de plantas Euphorbiaceae y Compositae por ser las que presentan un mayor número de relaciones con especies de sírfidos. Euphorbia serrata L. ha sido la planta sobre la que se ha registrado el mayor número (13) de especies de sírfidos (Figura 7). Por el contrario, sobre Asphodelus fistulosus L. y sobre Rosa sp., solo se ha registrado la presencia de Eristalis tenax (Linnaeus, 1758) y E. balteatus respectivamente. Esta última especie ha 
sido también observada (aunque no capturada) de manera reiterada, volando bajo la copa de especies arbóreas como Pinus sp., Ficus sp. y Brachychiton sp.

Una mayor biodiversidad vegetal va asociada generalmente a un mayor número de interacciones biológicas entre los insectos y las plantas. De este modo, se favorecen las funciones ecosistémicas como el control de plagas y la polinización y, por lo tanto, el equilibrio de estos ecosistemas. Existe un factor atrayente visual hacia las flores con colores amarillos, blancos y violáceos (Van Rijn \& Wäckers, 2016).

La visita de los sírfidos adultos a las flores se realiza constantemente, a lo largo del día y a un amplio rango de especies florales, por lo que su actividad polinizadora es complementaria y ocasionalmente tan importante como la que realizan los himenópteros (Doyle et al., 2020; Ssymank et al., 2008). Una de las razones que justifican la importancia de los sírfidos como agentes polinizadores en áreas urbanas y suburbanas como es el campus universitario, es que son más abundantes que muchas de las especies de himenópteros polinizadores, incluidas las abejas que son sus principales competidores en las visitas florales (Ssymank, 2003; Pérez Bañón et al., 2007). Por otra parte, los sírfidos carecen de veneno y son totalmente inofensivos, razón por la que algunas de sus especies se utilizan como polinizadoras en determinados cultivos bajo abrigo.

En las interacciones obtenidas entre sírfidopulgón presa, podemos ver que el mayor registro se da en especies vegetales no consideradas propiamente como ornamentales, sino como malas hierbas, como son las Euphorbiaceae y Asteraceae. Estas plantas se eliminan regularmente de las zonas ajardinadas y aledañas al campus, disminuyendo significativamente la biodiversidad de las especies de sírfidos estudiadas. Estudios anteriores ya habían demostrado que la biodiversidad de insectos en los parches de vegetación espontánea contiguas a las zonas ajardinadas o manejadas, representan un beneficio para el desarrollo heterogéneo del paisaje sin que suponga un coste económico adicional significativo (Montero, 2008). En nuestro estudio, se demuestra que estas plantas también actúan como refugio y sustento de especies controladoras de plagas cuyos adultos además son agentes polinizadores.
Con la información aportada, podemos concluir que la vegetación espontánea (las denominadas malas hierbas) del campus $(76,92 \%)$, así como las especies ornamentales $(12,82 \%)$ y hortícolas $(10,26 \%)$ estudiadas, presentan complementariedad en la compleja red de asociaciones tróficas establecida entre los dípteros Syrphidae, sus presas (Aphididae) y las plantas huéspedes de éstas.

No obstante, se trata de resultados establecidos durante 3 meses de muestreo, por lo que el número de asociaciones existentes es seguramente mayor, sabiendo que el complejo de especies espontáneas vegetales y de sírfidos asociados es dinámico y parcialmente cambiante con el transcurso de los meses del año. Estos datos son de utilidad en la mejora de las estrategias de gestión del campus relacionadas con el manejo de la vegetación silvestre y de actividades de jardinería como la poda o métodos de control de plagas que sean compatibles con la conservación de las especies polinizadoras y beneficiosas para el control biológico de plagas en las zonas ajardinadas de la Universidad de Alicante.

\section{AGRADECIMIENTOS}

A Antonio Ricarte, Zorica Nedeljković y Pablo Aguado por la identificación de algunas especies de sírfidos. A la Dra. Pilar Mier, especialista en taxonomía de áfidos, por su colaboración en la identificación de pulgones y al Dr. Segundo Ríos por su labor como botánico experto en la identificación de especies vegetales.

\section{REFERENCES}

Auad, A.M. (2003). Aspectos biológicos dos estágios imaturos de Pseudodorus clavatus (Fabricius) (Diptera: Syrphidae) alimentados com Schizaphis graminum (Rondani) (Hemiptera: Aphididae) em diferentes temperaturas. Neotropical Entomology, 32(3): 475-480. https://doi.org/10.1590/S1519-566X2003000300015

Brotons, J.C.A., Recuerda, A.G., García, J.L.V., Cabiedes, A.V., Lastres, P.R. \& Ruiz, S.R. (2009). Catálogo de las especies adventicias y ruderales del Campus de San Vicente del Raspeig, Universidad de Alicante. Bouteloua, (6): 87-100 (XI-2009). ISSN 1988-4257 
Costa, M. (1982). Pisos bioclimáticos y series de vegetación en el área valenciana. Cuadernos de geografía, (31): 129144.

Doyle, T., Hawkes, W.L.S., Massy, R., Powney, G.D., Menz, M.H.M. \& Wotton, K.R. (2020). Pollination by hoverflies in the Anthropocene. Proceedings of the Royal Society B, 287: 20200508. http://dx.doi.org/10.1098/ rspb.2020.0508

Hedo, E.B. (2012). Real Decreto 1311/2012, de 14 de septiembre, por el que se establece el marco de actuación para conseguir un uso sostenible de los productos fitosanitarios (BOE núm. 223, de 15 de septiembre de 2012). Actualidad Jurídica Ambiental, (17): 11-13.

Hernández-Martínez, K. \& Marcos-García, M. (2019). Entomofauna asociada a las plantas del campus de la Universidad de Alicante (España). Cuadernos de Biodiversidad, 57: 23-41. doi:10.14198/ cdbio.2019.57.03

Herrera, C.M. (1984). Tipos morfológicos y funcionales en plantas del matorral mediterráneo del sur de España. Studia Oecologica, 5(1): 7-34.

Hurtado, P. (2013). Estudio del ciclo de vida de sírfidos eristalinos (Diptera, Syrphidae) y bases para su cría artificial. Tesis doctoral Universidad de Alicante, España, 291pp.

Luna, J.M. (2005). Técnicas de colecta y preservación de insectos. Boletin Sociedad Entomológica Aragonesa, 37; 385-408. ISSN 1134-6094

Marcos-García, M.A. \& Galante, E. (2004). Métodos generales de preparación y conservación. En: Barrientos (ed.), Curso práctico de Entomología. Publicaciones Univ. Autónoma de Barcelona, CIBIO ediciones, Asociación española Entomología. España. ISSN 84-490-2383-1. pp. $47-54$.

Mexzón, R.G. \& Chinchilla M.C. (2003). Especies vegetales atrayentes de la entomofauna benéfica en plantaciones de palma de aceite (Elaeis guineensis Jacq.) en Costa Rica. Revista Palmas, 24(1): 33-57.

Montero, G. (2008). Bordes con vegetación espontánea en agroecosistemas pampeanos. Reservorios de plagas. Revista Agromensajes, 25: 25-30. ISSN 16698584 http://www.fcagr.unr.edu.ar/Extension/Agromensajes/2 5/9AM25.
Morales Marcos, I., Aguado Fernández, J.M., Nebreda Hernández, M., Díaz Desani, B., Romero, A., Pineda Gómez, A.M. \& Fereres Castiel, A. (2006). Diversidad de enemigos naturales de pulgones en cultivos de lechuga. Cuadernos de Biodiversidad, 21: 15-19. http:// dx.doi.org/10.14198/cdbio.2006.21.03

Pérez-Bañón, C., Petanidou, T. \& Marcos-García, M.A. (2007). Pollination in small islands by occasional visitors: the case of Daucus carota subsp. commutatus (Apiaceae) in the Columbretes archipelago, Spain. Plant Ecology, 192: 133-151. doi:10.1007/s11258-006-92331

Pineda, A. \& Marcos-García, M.A. (2008). Introducing barley as aphid reservoir in sweet-pepper greenhouses: Effects on native and released hoverflies (Diptera: Syrphidae). European Journal of Entomology, 105: 531535. doi:10.14411/eje.2008.070

Rojo, S., Gilbert, F., Marcos-García, M.A., Nieto, J.M. \& Mier, P. (2003). A world review of predatory hoverflies (Diptera, Syrphidae: syrphinae) and their prey. CIBIO (ed.), Spain, 219 pp. ISBN: 84-600-9854-0

Rotheray, G.E. \& Gilbert F. (2011). The Natural History of Hoverflies. Ceredigion, UK: Forrest Text, 333 p. ISBN: 978-0-9564692-1-2

Sommaggio, D. (1999). Syrphidae: can they be used as environmental bioindicators? Agriculture, Ecosystems and Environment 74: 343-356. https://doi.org/10.1016/ S0167-8809(99)00042-0

Ssymank, A. (2003). Habitatnutzung blütenbesuchender Schwebfliegen (Diptera, Syrphidae) in Wald-OffenlandVegetationsmosaiken. Berichte der Reinhold-TüxenGesellschaft, 15: 215-228.

Ssymank, A., Kearns, C.A., Pape, T. \& Thompson, F.C. (2008). Pollinating Flies (Diptera): A major contribution to plant diversity and agricultural production. Biodiversity, 9(1-2): 86-89. doi:10.1080/1 4888386.2008.9712892

Van Veen, M.P. (2004). Hoverflies of Northwest Europe. Identification Keys to the Syrphidae. KNNV Publishing, Utrecht, 254 pp. https://doi. org/10.1163/9789004274495

Van Rijn, P.C.J. \& Wäckers, F.L. (2016). Nectar accessibility determines fitness, flower choice and abundance of hoverflies that provide natural pest control. Journal of Applied Ecology, 53: 925-933. https://doi. org/10.1111/1365-2664.12605 